\title{
Parametrization of electromechanical systems must acknowledge Newton and Maxwell
}

\author{
Natasha Hirschfeldt ${ }^{1}$ \\ PUC-Rio, Rio de Janeiro, RJ \\ Roberta Lima ${ }^{2}$ \\ PUC-Rio, Rio de Janeiro, RJ \\ Rubens Sampaio ${ }^{3}$ \\ PUC-Rio, Rio de Janeiro, RJ
}

\begin{abstract}
Electromechanical systems are an interesting type of coupled systems. They are composed by two subsystems with different nature: mechanical and electromagnetic. The subsystems interact. To represent the dynamics of a coupled system, it is necessary to properly characterize their interaction. The dynamics of an electromechanical system is given by an initial value problem (IVP) comprising a set of coupled differential equations involving, necessarily, mechanical and electromagnetic variables. Despite the ubiquity of electromechanical systems, a few authors do not parametrize them properly. Frequently, by some artifice, strange to the problem, the coupled system is uncoupled disregarding the electromagnetic subsystem. Hence, the uncoupled system has a different dynamics, resulting a reduced IVP with only a mechanical equation. This paper discusses this uncoupling using as example a galvanometer, a well-known measuring device. To analyze the effects of the decoupling, numerical simulations of the two IVP, complete and reduced, are performed.
\end{abstract}

Key words. Lagrangian, Energy, Co-energy, Electromechanical system, Galvanometer.

\section{Introduction}

Electromechanical systems are composed by two coupled subsystems: a mechanical and an electromagnetic $[1,10,12]$. To properly describe the dynamics of an electromechanical system, it is not sufficient to describe the dynamics of each subsystem separately. It is necessary to characterize the interaction between them. The dynamics is given by an initial value problem (IVP) comprising a set of coupled differential equations. This set involves mechanical and electromagnetic variables and initial conditions. The dynamics of an electromechanical system can be found by the Lagrange method, an energetic method. The Lagrange function must have a term that couples the mechanical and the electromagnetic subsystems and creates a energy flux between them $[2,3]$.

Even though electromechanical systems are found in several applications, it is still a challenge to find references correctly describing their dynamics. Published papers, books and thesis present numerous mistakes in the formulation of the dynamics of this type of system. They uncouple the mechanical and an electromagnetic subsystems by neglecting the existence of inductance in the electromagnetic subsystem and assuming a functional relation between mechanical and electromagnetic variables. Therefore, the system is characterized by only mechanical variables and the

\footnotetext{
${ }^{1}$ natashaboh97@gmail.com

${ }^{2}$ robertalima@puc-rio.br

3 rsampaio@puc-rio.br
} 
dynamics is reduced to an IVP with only a mechanical equation [4-8,11]. Some references that makes the reduction are references 1,2 and 3 of [6] and reference 4 of [5].

Apparently, the reduction is useful, since it simplifies the dynamics greatly. The reduced IVP is easier to handle. However, the reduction changes the dynamics. The reduced IVP does not represent the complete system and moreover, decouples the electromagnetic and mechanical subsystems. The dynamics of the electromagnetic subsystem is ignored and the space phase is maimed (it just has the variables of the mechanical subsystem). The paper's main objective is to show that reduction changes the dynamics using as example a galvanometer, a measuring device.

This paper is organized as follows. Section 2 describes the galvanometer and the mechanical and electromagnetic variables used to characterize the system state. In Section 3, the galvanometer's dynamics is found by Lagrange method. In Section 4, the reduced IVP with only a mechanical equation is presented. To compare and verify the differences between the complete and reduced dynamics, numerical simulations of the two IVP were performed using the software MATLAB. The results are shown in Section 5 and the conclusions in Section 6.

\section{Galvanometer}

A galvanometer is an electromechanical system used to measure electric currents with low intensities. It is composed by a circuit with inductance $l$, resistance $r$, a permanent magnet, torsion springs with stiffness $k$, a damper $b$, a fix iron core with height $L$, diameter $2 d$ and moment of inertia $j$, a coil involving the core and a pointer connected to the core. The coil has many turns and it is pivoted on a bearing. It can rotate in the magnetic field produced by the magnet. The generalized variables used to represent the system dynamics are the current $\dot{q}$ and the pointer's angle $\theta$. It is not taken into account capacitance in the system. The system is sketched in Figure 1.

When the galvanometer is submitted to a voltage $v$, a current, $\dot{q}$, is generated in the circuit of the system. When this current passes through the moving-coil, creates a magnetic field $B$. The interaction between this induced field and the field produced by the magnet produces a torque in the coil. This causes the coil to rotate and the pointer to deflect over a graduated scale. The torques generated by torsion spring and damper, mounted on the bearing and coiled in opposite direction, balance the electromagnetic torque.

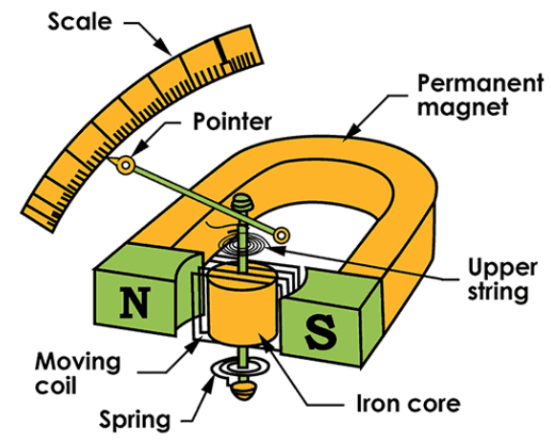

Figure 1: Galvanometer [9].

\section{Lagrangian formulation for a galvanometer}

The Lagrangian function for an electromechanical system [10] is written as: 


$$
\Gamma=T^{*}-V+E_{m}^{*}-E_{e} \pm U^{*},
$$

where $T^{*}$ is the kinetic co-energy, $V$ the potential energy, $E_{m}^{*}$ the magnetic co-energy and $E_{e}$ the electric energy. The coupling term $U^{*}$ can have an electric or magnetic origin and it's signal depends on this fact. If it is transmitted as a magnetic coupling $\left(U_{m}^{*}\right)$, the signal is positive and if it is transmitted as an electric one $\left(U_{e}^{*}\right)$, the signal is negative.

The modulus of the electromagnetic torque generated over the coil is $\tau_{B}=\dot{q} B d L$, where $B$ is the magnetic field considered constant. Representing the constant $B d L$ as $\gamma$ and the magnetic co-energy is then given by $U_{m}^{*}=\gamma \dot{q} \theta$.

The other energies in the system are $T^{*}=\frac{j \dot{\theta}^{2}}{2}, V=\frac{k \theta^{2}}{2}, E_{e}=0, E_{m}^{*}=\frac{l \dot{q}^{2}}{2}$. Therefore, the Lagrangian function for the galvanometer is

$$
\Gamma=\frac{j \dot{\theta}^{2}}{2}-\frac{k \theta^{2}}{2}+\frac{l \dot{q}^{2}}{2}+\gamma \dot{q} \theta
$$

where $\Gamma$ is given in joules. The units of $j$ is kilogram per meter squared, of $k$ is newton per meter, $l$ is given in henry and the unit of $\gamma$ is meter squared tesla. Also, the variables $\theta, \dot{\theta}$ and $\dot{q}$ are given in radians, radians per seconds and amperes, respectively.

The virtual work is:

$$
\delta_{f}=v \delta q, \quad \delta_{d}=r \dot{q} \delta q+b \dot{\theta} \delta \theta \quad \rightarrow \quad \delta W=\delta_{f}-\delta_{d}=v \delta q-r \dot{q} \delta q-b \dot{\theta} \delta \theta .
$$

The dynamics equations can be found doing

$$
\left\{\begin{array}{l}
\frac{d}{d t}\left(\frac{\partial \Gamma}{\partial \dot{\theta}}\right)-\frac{\partial \Gamma}{\partial \theta}=\frac{d \delta W}{d \delta \theta} \\
\frac{d}{d t}\left(\frac{\partial \Gamma}{\partial \dot{q}}\right)-\frac{\partial \Gamma}{\partial q}=\frac{d \delta W}{d \delta q} .
\end{array} .\right.
$$

The IVP that gives the galvanometer dynamics is: given a source voltage $v$, find $(\theta, \dot{q})$ such that, for all $t>0$,

$$
\begin{cases}j \ddot{\theta}+b \dot{\theta}+k \theta-\gamma \dot{q} & =0 \\ l \ddot{q}+r \dot{q}+\gamma \dot{\theta} & =v\end{cases}
$$

with the initial conditions $\dot{\theta}(0)=\omega_{0} \mathrm{rad} / \mathrm{s}, \theta(0)=\theta_{0}$ rad and $\dot{q}(0)=c_{0}$ A.

\section{Reduced IVP with only a mechanical equation}

In this section we will discuss an artifice used in many references that deals with electromechanics systems. This artifice, apparently useful, simplifies the system dynamics. It reduces the IVP that gives the dynamics of an electromechanical system into a simpler IVP with only mechanical variables. However, the reduction causes the uncoupling of the mechanical and electromagnetic subsystems. After the reduction, the system becomes a purely mechanical system. In the case of the galvanometer, the reduction is done neglecting the existence of the inductance in the electromagnetic subsystem and assuming a functional relation between the mechanical and electromagnetic variables, $\theta$ and $\dot{q}$.

Neglecting the existence of the inductance in the second equation of the IVP given in (4), it is possible to write 


$$
\dot{q}=\frac{v-\gamma \dot{\theta}}{r},
$$

where $\dot{q}$ is given in amperes. Eq. (5) gives a functional relation between $\dot{q}$ and $\theta$. Substituting this functional relation in the second differential equation of the complete IVP, we obtain a reduced IVP with only mechanical variables and initial conditions. Given a constant source voltage $v$, find $\theta$ such that, for all $t>0$,

$$
\ddot{\theta}+\left[b+\frac{\gamma^{2}}{r j}\right] \dot{\theta}+\left[\frac{k}{j}\right] \theta=\left[\frac{\gamma}{r j}\right] v,
$$

with the initial conditions $\dot{\theta}(0)=\omega_{0} \mathrm{rad} / \mathrm{s}, \theta(0)=\theta_{0}$ rad. The current $\dot{q}$ can be computed after the reduced IVP is solved by Eq. (5).

Calling $\xi=\frac{r b+\gamma^{2}}{2 r \sqrt{k j}}, \omega_{n}=\sqrt{\frac{k}{j}}, p=\frac{\gamma}{k r}$, Eq. (6) can be rewritten as:

$$
\ddot{\theta}+2 \xi \omega_{n} \dot{\theta}+\omega_{n}^{2} \theta=\omega_{n}^{2} p v .
$$

The solution of the reduced IVP is given by

$$
\theta_{\text {reduced }}(t)=e^{-\xi \omega_{n} t}\left[\left(\theta_{0}-p v\right) \cos \left(w_{d} t\right)+\left(\frac{\omega_{0}+\xi w_{n}\left(\theta_{0}-p v\right)}{w_{d}}\right) \sin \left(w_{d} t\right)\right]+p v[r a d] .
$$

Then, it is possible to write

$$
\begin{array}{r}
\dot{\theta}_{\text {reduced }}(t)=-\xi w_{n} e^{-\xi \omega_{n} t}\left[\left(\theta_{0}-p v\right) \cos \left(w_{d} t\right)+\left(\frac{\omega_{0}+\xi w_{n}\left(\theta_{0}-p v\right)}{w_{d}}\right) \sin \left(w_{d} t\right)\right]+ \\
e^{-\xi \omega_{n} t}\left[-w_{d}\left(\theta_{0}-p v\right) \sin \left(w_{d} t\right)+\left(\omega_{0}+\xi w_{n}\left(\theta_{0}-p v\right)\right) \cos \left(w_{d} t\right)\right],
\end{array}
$$

Here, $\theta_{\text {reduced }}(t)$ is given in radians and $\dot{\theta}_{\text {reduced }}(t)$, in radians per seconds.

\section{Comparison between the complete and reduced IVP}

A routine was developed in the software $M A T L A B$ to simulate the complete and reduced IVP. Comparing the system response over time and phase portraits for the two IVP, it is possible to see how the reduction changes the dynamics.

The complete IVP was integrated by the $4^{\text {th }}-5^{\text {th }}$ order Runge-Kutta method with the ode 45 $M A T L A B$ function. The time-step used was 0.002 seconds and the time interval $[0,200]$ seconds. The parameters used in all simulations, chosen to highlight the effects of the uncoupling, are $j=0.50 \mathrm{~kg} / \mathrm{m}^{2}, b=0 \mathrm{Ns} / \mathrm{m}, k=0.20 \mathrm{~N} / \mathrm{m}, \gamma=1.00 \mathrm{~m}^{2} \mathrm{~T}, l=10.00 \mathrm{H}, r=10.00 \Omega$ and $v=1 \mathrm{~V}$.

To analyze the influence of initial conditions in the system response, two groups of values were selected.

- Case I: null initial conditions for the complete and reduced IVP.

- Case II: $\theta(0)=1 \mathrm{rad}$ for the complete and reduced IVP and zero for all others initial conditions. 


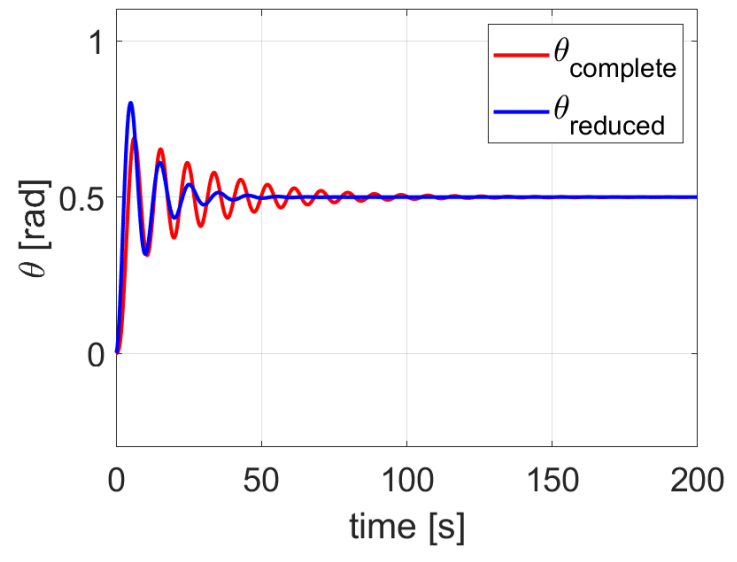

(a)

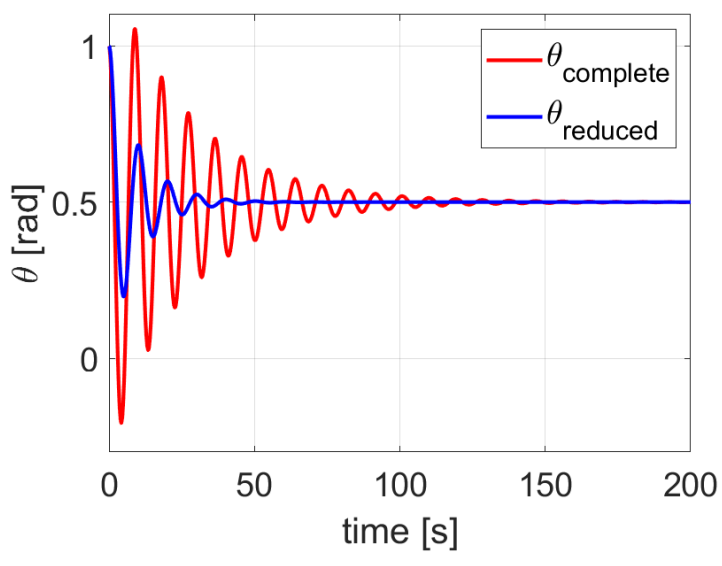

(b)

Figure 2: (a) Angle $\theta$ over time for complete and reduced dynamics for case I and (b) case II.

Figure 2 shows $\theta(t)$ over time for the complete and reduced IVP, red and blue curves respectively, for cases I and II. Please observe that both curves tend to the same value, $0.5 \mathrm{rad}$, when $t$ grows. However the damping factors are distinct. The reduced IVP is more damped than the complete one.

Figures 3(a) and 3(b) show the phase portraits of the variables $\dot{\theta}$ and $\dot{q}$ for the complete and reduced IVP. Observing the results, one sees immediately the differences between the results. While for the complete IVP, there is no functional relation between $\dot{\theta}$ and $\dot{q}$, for the reduced IVP, there is a functional relation between $\dot{\theta}$ and $\dot{q}$. This functional relation is given by Eq. (5). The lack of a functional relation is the essence of coupling.

Comparing Figures 3(a) and 3(b) it is possible to verify also that influence of the initial conditions in the response of the complete and reduced IVP. For the reduced IVP, the change of initial conditions from case I to case II causes a small modification in the phase portraits of the variables $\dot{\theta}$ and $\dot{q}$. On the other hand, for the complete IVP, the change of initial conditions from case I to case II modifies a lot the phase portraits. The red curve of Figure 3(a) is very different from the red curve of Figure 3(b).

Figures 4(a) and 4(b) show the phase portraits of $\tau$ and $\dot{\theta}$. Please observe again the functional relation between $\tau$ and $\dot{\theta}$ in the reduced IVP.

\section{Conclusions}

Electromechanical systems are composed by two subsystems with different origins: mechanical and electromagnetic. The dynamics of an electromechanical system is given by an initial value problem with coupled differential equations. These equations involve mechanical and electromagnetic variables.

This paper analyzes a common error found in the literature that deals with electromechanical systems. Here, the error is discussed in the context of an galvanometer, but it the literature, the error appears in many different contexts $[4-7,11]$. The error is an artifice to simplify the system dynamics. It reduces the IVP that gives the dynamics of an electromechanical system into a simpler IVP with only mechanical variables. After the reduction, the system becomes a purely mechanical system. Apparently, the reduction is useful, but it changes the dynamics. 


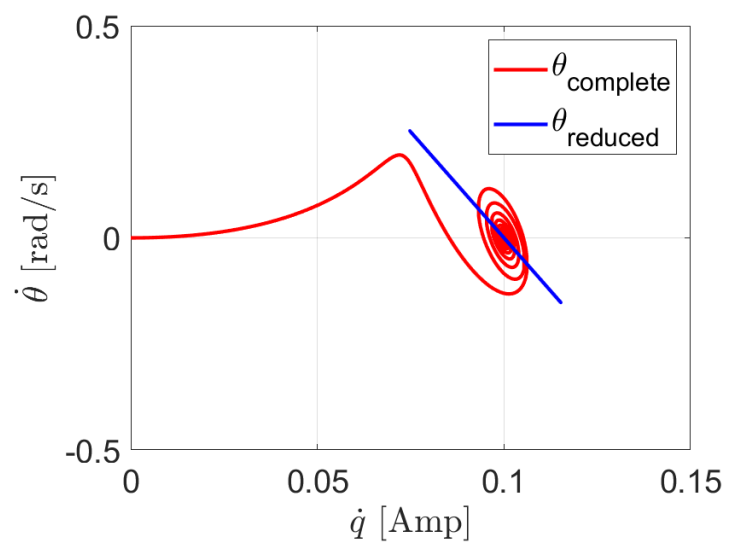

(a)

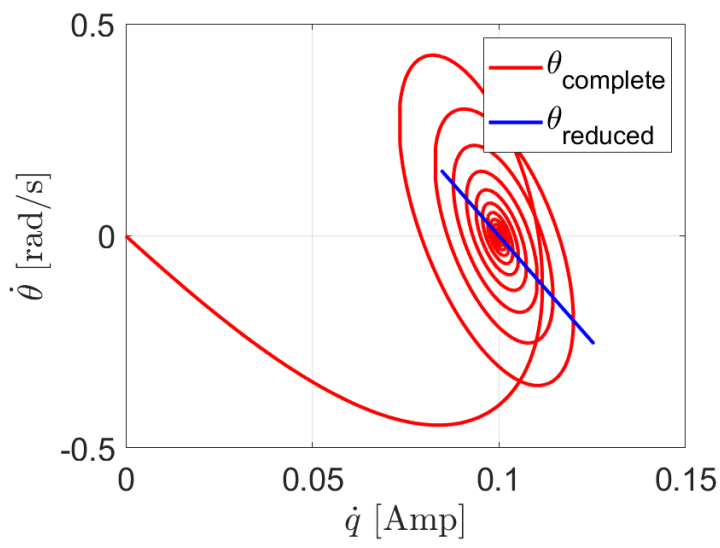

(b)

Figure 3: (a) Phase portrait of $\dot{\theta}$ and $\dot{q}$ for case I and (b) case II.

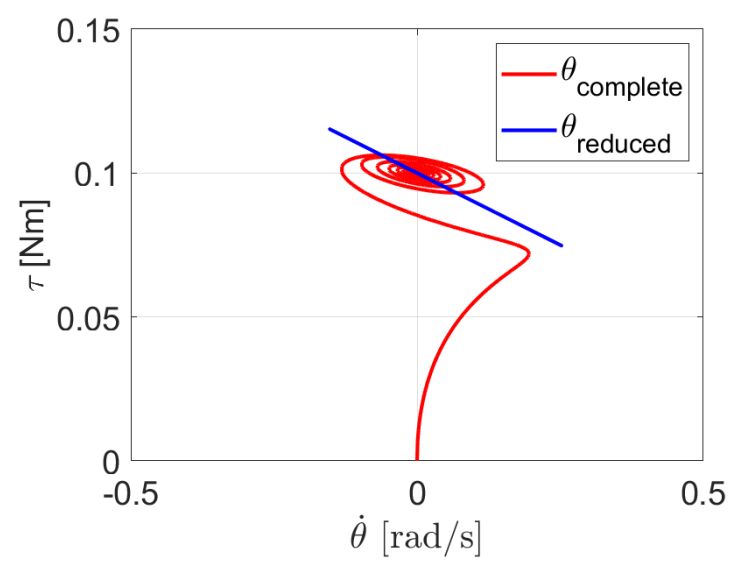

(a)

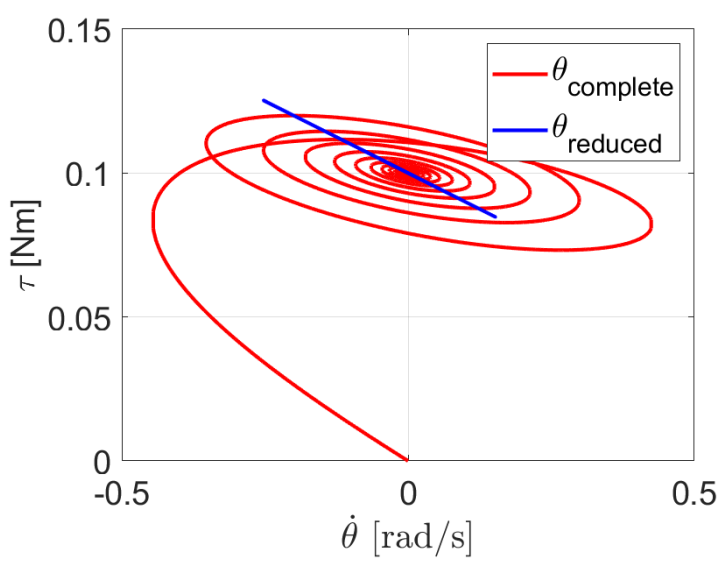

(b)

Figure 4: (a) Phase portrait of $\tau$ and $\dot{q}$ for case I and (b) case II.

\section{Acknowledgments}

The authors acknowledge the support given by FAPERJ, CNPq and CAPES.

\section{References}

[1] Jeltsema, D. and Scherpen, J. M. A. Multidomain modeling of nonlinear networks and systems. IEEE Control Systems, volume 29, no. 4, pages 28-59, 2009. DOI: 10.1109/MCS.2009.932927.

[2] Hirschfeldt, N., Lima, R. and Sampaio, R. Coupling in electromechanical systems, Encontro Regional de Matemática Aplicada e Computacional (ERMAC-MS), 2020.

[3] Hirschfeldt, N., Lima, R. and Sampaio, R. Electromagnetic loudspeaker: an energetic approach, XIV Encontro Acadêmico de Modelagem Computacional, 2021. 
[4] Lima, R. and Sampaio, R. Two parametric excited nonlinear systems due to electromechanical coupling. Journal of the Brazilian Society of Mechanical Sciences and Engineering, volume 38, pages 931-943, 2016.

[5] Lima, R. and Sampaio, R. Pitfalls in the dynamics of coupled electromechanical systems. CNMAC 2018, Proceeding Series of the Brazilian Society of Computational and Applied Mathematics, 2019.

[6] Lima, R., Sampaio, R., Hagedorn, P. and Deü, J. Comments on the paper "On nonlinear dynamics behavior of an electro-mechanical pendulum excited by a nonideal motor and a chaos control taking into account parametric errors" published in this journal. Journal of the Brazilian Society of Mechanical Sciences and Engineering, volume 41, page 552, 2019.

[7] Manhães, W., Sampaio, R., Lima, R., Hagedorn, P. and Deü, J. Lagrangians for electromechanical systems. Mecánica Computacional, volume XXXVI, pages 1911-1934, 2018.

[8] Manhães, W., Sampaio, R., Lima, R. and Hagedorn, P. Two coupling mechanisms compared by their Lagrangians. DINAME 2019, Proceedings of the XVIII International Symposium on Dynamic Problems of Mechanics, 2019.

[9] Madhu. Moving Coil Meter. Codrey Electronics, 26, June, 2020. Available: < https://www.codrey.com/electrical/moving-coil-meter/ >. Access in: 13, March, 2021.

[10] Preumont, A. Mechatronics: dynamics of electromechanical and piezoelectric systems, volume 136, G.M.L. GLADWELL, University of Waterloo, Canada, 2006.

[11] Sampaio, R., Lima, R. and Hagedorn, P. One alone makes no coupling. Mecánica Computacional, volume XXXVI, pages 931-944, 2018.

[12] Wells, D. A. Schaum's outline of theory and problems of Lagrangian dynamics with a treatment of Euler's equations of motion, Hamilton's equations and Hamilton's principle, New York: McGraw-Hill, 1967. 